\title{
Closing remarks and Outlook
}

\author{
Francoise Combes ${ }^{1}$ \\ ${ }^{1}$ Observatoire de Paris, LERMA, College de France, CNRS, PSL, Sorbonne Univ. UPMC, \\ F-75014, Paris, France \\ email: francoise.combes@obspm.fr
}

\begin{abstract}
Some highlights are given of the IAU Symposium 334, Rediscovering our Galaxy, held in Potsdam, in July 2017: from the first stars fossil records found in the halo, the carbonenhanced metal poor CEMP-no, to the cosmological simulations presenting possible scenarios for the Milky Way formation, passing through the chemo-dynamical models of the various components, thin and thick disks, box/peanut bulge, halo, etc. The domain is experiencing (or will be in the near future) huge improvements with precise and accurate stellar ages, provided by astero-seismology, precise stellar distances and kinematics (parallaxes and proper motions from GAIA), and the big data resulting from large surveys are treated with deep learning algorithms.
\end{abstract}

Keywords. Galaxy: abundances - Galaxy: bulge - Galaxy: disk - Galaxy: evolution - Galaxy: halo - Galaxy: kinematics and dynamics - Galaxy: structure - stars: abundances

Due to our location well inside the plane, far from the center, the image we have of our Galaxy is quite indirect, highly dependent on models, and inspired by potentially similar external galaxies. Frequently along this week, artist's views of the face-on Milky Way were shown (often from R. Hurt), but some have two arms and a bar, some have four arms, more or less winding out. So many different points of view appeared, that it could remind us the blind scientists examining an elephant, and finding a fan, wall, tree, snake or rope.

\section{Fossil records and halo stars}

The oldest stars, found in the stellar halo, are extremely metal poor, only enriched by the first stars in the Universe, the PopIII stars which have already disappeared. They are rare, with peculiar abundances. Most of them are carbon enriched and are dubbed CEMP stars (cf J. Norris review talk).

One of the main impact of rotation in massive stars is to produce nitrogen, carbon and some s-process already in the first phases of the chemical enrichment of the Universe. As Georges Meynet emphasized in his beautiful talk, rotation is particularly important for massive low-metallicity stars, since they lack of opacity. Without rotation, they would only have little stellar winds or mixing. Rotation helps the elements formed in the stellar core to come to the surface. For solar-metallicity stars on the main sequence, rotation makes very little difference. Fast-rotating massive stars, or spinstars have been modeled successfully and can explain the abundances of CEMP stars (Meynet et al 2006, 2010).

The neutron-capture elements (Sr first peak, Ba second peak) are also peculiar, they might be formed by s or $\mathrm{r}$ process. However, most of the CEMP-s and r/s stars are binaries, their peculiar abundances can be explained by mass transfer from an AGB star to a lower mass companion. Eventually, the CEMP-no stars are the best tools for galactic archeology.

Now we learn that these CEMP stars exist also in dwarfs (previously only in massive 
galaxies). The r-process elements, formed in rare explosive events, are particularly overabundant in Ret II, and only stochastic processes can account for that (cf the Sculptor dwarf and talk by A. Chiti).

The enrichment of old stars was detailed in G. Cescutti talk: models of supernovae scenarios with electron capture (EC) or Magneto-rotation driven (MRD) process were tested. The observed stochasticity is well reproduced. The abundance ratios between light $(\mathrm{Sr} \& \mathrm{Y}$ ) over heavy (Eu \& Ba) elements require spinstars (Cescutti \& Chiappini 2014). It might be possible to disentangle the various processes, through the $\mathrm{Ba}$ isotopic abundances.

The stellar halo is also very precious to learn about all accreting events experienced by the MW. Accreted dwarf companions can be followed through tidal streams and substructures in the halo. In her talk, A. Helmi demonstrated that simulated models are compatible with the whole halo assembled from companions. There are however some discrepancies between the $[\alpha / \mathrm{Fe}]-[\mathrm{Fe} / \mathrm{H}]$ abundances of halo stars and those of dwarfs today, so not all the stellar halo could come from this kind of dwarfs. There could exist two components in the stellar halo, i.e. an inner \& outer halo (e.g. Carollo et al., 2016).

The observation of the tidal streams (with TGAS+RAVE) is essential to characterize the dark matter halo. Several studies have not converged yet on its flattening. Certainly there is hope in the near future from the proper motions, that will be determined with high precision by GAIA. In her talk N. Kallivayalil updated her measurement of the LMC proper motions, and the coherence and accuracy was impressive. This was crucial to change our view of the Magellanic Clouds orbits, which are now known to be on their first passage around the Milky Way.

\section{Amplification from gravitational lenses}

In his talk, T. Bensby described how useful were lenses to study high resolution spectra of dwarf stars in the bulge, with high signal to noise ratio. Without gravitational amplification, it would not have been possible. Several episodes of star formation have been identified: $3,6,8$, and $11 \mathrm{Gyr}$ ago in the bulge. The spectra show several peaks in $[\mathrm{Fe} / \mathrm{H}]$ over an otherwise wide metallicity distribution. For the low metallicity bulge stars, the knee of $[\mathrm{alpha} / \mathrm{M}]$ appears at higher $[\mathrm{Fe} / \mathrm{H}]$ than for the local thick disk. However, the finding of so many young stars in the bulge is in contradiction with HST proper motion cleaned CMDs, and with the fact that a counterpart of this younger population has not yet been found. Some biases, or contamination by foreground are possible.

Micro-lenses from OGLE were also used by Wegg et al. (2017) to characterize stellar populations towards the bulge. With 3000 micro-lensing events, it was possible to constrain the masses of the lenses, since they determine the time-scale of lensing. Together with the M2M dynamical model, done with O. Gerhard group, it was possible to show that the stellar IMF (essentially its low-mass end) is constant in the Galaxy, whatever the age of the populations, or the location (bulge, disk), or the alpha over-abundance.

C. Wegg also discussed the constraints on the dark matter profile that can be derived. It is pleasant to see that the micro-lensing experiments, that were unable to find the dark matter in compact objects they were searching for, now succeed eventually to derive constraints on the dark matter halo of the Milky Way. The stellar masses in the center constrain the $\mathrm{M} / \mathrm{L}$ of the baryons, and a more accurate dark matter density is obtained. Together with the rotational velocity constraints at the solar circle, this leads to the conclusion that there must exist a large core in the dark matter distribution. 


\section{Abundance surveys of the Milky Way}

Chemical tagging is a precious tool to disentangle the history and formation of the various components of the Milky Way. Doubts of its reliability were discussed by R. Smiljanic in his talk, since already large abundance inhomogeneities can be observed in a given open cluster. However, the thin and thick disks components can be easily identified in many surveys (RAVE, APOGEE, GALAH, GES, LAMOST in outer parts, etc.). Convincing results were shown by J. Holtzman in his talk. In particular the $[\alpha / \mathrm{Fe}]$ versus $[\mathrm{Fe} / \mathrm{H}]$ plots at various radii and heights above the plane by Hayden et al. (2015) have been viewed several times during the week, and they are very encouraging. They show that indeed chemical tagging is possible, since the alpha versus metallicity diagrams separate very clearly the thick and thin disk, in agreement with their locations.

D. Minniti presented the survey of variable stars VVV $=$ VISTA Variables in the Va Lactea. It is a public ESO variability survey in the near-infrared of RRLyrae, Cepheids, and Red Clump stars. Already 1 billion sources have been studied, over 562 square degrees.

The metallicity maps, decomposed in metal-poor and metal-rich components, reveal clearly the bulge/thick disk (very compact component, with short characteristic radius) and metal-rich disk, with large characteristic radius. Zoccali et al 2017 (GIBS survey) show clearly the bimodality between these two metallicities.

Many publications have already appeared on the GAIA-ESO survey; A. Recio-Blanco described their rich results in her review talk, embellished by a personal movie. I will just highlight one of them, the discovery of low [alpha/M] abundance in some bulge stars, which have similar chemical properties with the thin disk. This confirms the complex multi-component character of the bulge (Recio-Blanco et al 2017).

Annie Robin presented an update of the Besançon local model with TGAS+RAVE. This updated model is based on Stäckel potentials, and an asymmetric drift in 3D. The chemical properties of the thick and thin disks are taken into account. Good agreement is obtained with observations, also for the kinematics, although a small $\mathrm{V}$ value for the Sun motion is derived (Robin et al 2017).

\section{Chemo-dynamical models of the Milky Way}

In her talk, Lia Athanassoula convinced us that disks can form after a major merger (Athanassoula et al 2016). This is a possible scenario for the Milky Way, if the major merger event occurred more than 8 Gyr ago. In their chemodynamical models (Athanassoula et al. 2017) the mono-abundance populations succeed in reproducing observations.

Chemo-dynamical models of the barred inner galaxy were also described by Ortwin Gerhard, who showed a point of view corresponding to some convergence among several dynamical works (e.g. Di Matteo et al. 2014, Debattista et al. 2017): The classical bulge is very limited ( $<5 \%$ in mass), most prominent are the thick and thin disks, forming a box/peanut bulge. Important are also the effects of radial migration, mapping the outer disk in the upper box/peanut component. With these ingredients, it is possible to explain the vertical and radial abundance gradients observed in the Milky Way.

In particular a recent model by Fragkoudi et al. 2017 (presented in poster 20) showed how a thin+thick disk model succeeds in reproducing the positive abundance gradient with longitude. Previously, it was thought that only a negative gradient of a thin disk, then combined with radial migration and formation of a box/peanut bulge, was sufficient to create this positive gradient. In recent publications, Portail et al (2017a,b) succeeded to reproduce the observations, with a made-to-measure model (M2M) and chemical tagging. 
The reversal of abundance gradient at high latitude, attributed by Minchev et al. (2014) to flaring of mono-age disk populations, has been confirmed in simulations by D. Kawata to be due to a flaring of the thin disk at large radii. In the plane, the abundance gradient is negative. But at large height $\mathrm{z}>2 \mathrm{kpc}$ above the plane, it becomes positive. Since the thick disk disappears relatively quickly with radius, the abundance corresponds then to the thin disk, which becomes thick, and makes the continuity with the thick disk at large radii (Rahimi et al. 2014).

This interpretation is confirmed with an age gradient study, as presented by M. Martig in her talk. Comparing the radial distribution of age and $[\alpha / \mathrm{Fe}]$ shows that alpha/M is indeed a relative indicator of age. While age decreases with radius and increases with height above the plane, it converges at large radius and height to values comparable to that of the thin disk (Martig et al. 2016).

It is possible that several mono-age populations are each flaring, nested in each other. This will follow the inside-out formation of the thin disk. These multiple flaring disks, at progressively larger radius, might explain the observed constant stellar scale-height of external edge-on disks (Minchev et al. 2015).

More generally, to reconstruct the formation of our Galaxy, it is primordial to identify age populations. A proxy has been to use $[\mathrm{Fe} / \mathrm{H}]$ and $[\alpha / \mathrm{Fe}]$, together with other abundances, to identify Mono-Abundance populations (MAP), which might be also mono-age population. But there are large uncertainties (Minchev et al. 2016). And even young $[\alpha / \mathrm{Fe}]$ stars have been found (Chiappini et al. 2015, Martig et al. 2015). In the combined sample CoRoGEE (CoRoT + APOGEE), where ages are better defined with asteroseismology masses, there is indeed a reversal of age populations, that radial migration is insufficient to explain (Anders et al. 2017a,b).

These young $[\alpha / \mathrm{Fe}]$ star could have been formed from gas enriched by thick disk stars, they will constitute an intermediate thick disk. Alternatively they might not be really young, but evolved blue stragglers from the old thick disk population (Jofré et al. 2017).

\section{Ages of stars}

Marcio Catelan reviewed all methods to obtain the ages of stars (see also Soderblom 2010), in particular nucleo-cosmochronometry, and the isochrone methods, based on the main sequence turn off (MSTO) which correspond to imperfect clocks, although they are still valuable to find relative ages, as Haywood et al. (2013) or Bensby et al. (2014) have shown. In the near-future, there will be large improvements with GAIA and asteroseismology (CoRoT, Kepler, K2), see the workshop Chiappini, Montalban \& Steffen (2016).

In the models, there are frequently stars older than the Universe; about 20 yrs ago, this was solved by the introduction of dark energy or a cosmological constant. Today, CMB observations and the Planck collaboration come up with a high precision age of $13.802 \pm 0.026$ Gyr. Could stellar physics compete with this high precision cosmology? At present errors on stellar ages are still of the order of Gyr!

But there are bright new perspectives with asteroseismology, which provide accurate stellar masses, and help to raise degeneracies. Asteroseismology can be miraculous, when individual frequencies $\nu$ i can be determined, as Arlette Noels demonstrated in her talk. When only physical parameters such as L, Teff, Fe/H are known for stars on the main sequence (MS) or red giants $(\mathrm{RG})$ or red clump (RC), ages are known with $>80 \%$ uncertainty. When $\Delta \nu$ and $\nu \max$ are known from asteroseismology, these uncertainties become $>30 \%$ (MS), and $20 \%$ (RG and RC), but when the individual frequencies $\nu$ i are known, these become 20\% (MS) and 10\% (RG and RC). With Plato, it will be possible to 
follow up oscillations over large periods of time (e.g. 150 days) and $\nu$ i will be determined. Masses could be determined with $1 \%$ accuracy and ages with $3 \%$, as presented in G. Davies' talk (see e.g. Miglio et al. 2017).

Accurate ages will improve the determination of star formation histories (SFH). There has been several SFH determinations, based on age proxies such as $[\mathrm{Si} / \mathrm{Fe}]$ in the solar neighborhood, and then computation of chemical models for the whole Galaxy: stellar yield, mass loss are taken into account, together with the assumption that the inner disk operates in a quasi closed box model (Haywood et al. 2013, Snaith et al. 2014, 2015). These studies have concluded to the existence of a quenching episode 8 Gyr ago. SF quenching around $10 \mathrm{Gyr}$ ago is also the main conclusion of the two-infall model of Chiappini et al. (1997), or see also Fuhrmann (2011). This quenching could be due to a merger, or to the strengthening of a bar (or both). Bar quenching has been studied by Khoperskov et al. (2017, see poster 42).

E. Bernard presented a different method to obtain SFH, the global population method, based on CMD fitting, from GAIA + TGAS. The depth of the samples is ensured by the combination of information from Tycho2, Hipparcos, APASS. Of course these estimations will be much improved with the DR2 release of GAIA in April 2018. A preliminary SFH in the solar neighborhood shows very young stars in the recent 2-4 Gyr, following a rather slow star formation in the old ages. Towards Baade's window in the bulge, the SFH reveals the contrary: a starburst in the old time, and a rather quiescent bulge after $\mathrm{t}=8$ Gyr. This has been built directly from CMD, and radial migration has not yet been taken into account.

In his talk, F. Anders demonstrated the existence of 2 thick disks in the Milky Way. To deal with the large sample of APOGEE-TGAS, the t-SNE method was used for the first time in abundance space, to take into account 15 chemical abundances. The method was applied on an alpha/abundance diagram, weeded out from blurring migrating stars (those with high excentricity). A third component clearly stands out, the "hamr": high alpha metal rich thick disk. This could mean that the formation of thick and thin disks overlap in time. Radial migration might also have an effect there.

\section{Gravitational potential of the Milky Way: dark matter}

J. Binney discussed in his talk about the dynamical methods to model accurately the Milky Way in the GAIA era. Some assumptions have to be made, of a relaxed body, possessing some symmetries; all non-axisymmetries will then be dealt with as perturbations. The method decomposes the Milky Way into multiple components according to age, chemical composition, kinematics, geometry (thin and thick disks, bulge). The modeling is based on action variables, and at each component corresponds a function $\mathrm{f}(\mathrm{J})$ of the integral of motions. Detailed agreement is obtained with local data RAVE-TGAS (e.g. Binney 2017).

H-W. Rix was less optimistic, and also proposed symmetric models, where bars, dark matter halo flattening and satellites would be treated as perturbations. However, we must admit that the present tidal streams studies have not yet given clues for the shape of the dark matter halo. Certainly, it is difficult to assume that these tidal streams are relaxed, and in equilibrium in the potential. It is not sure that more and better data will improve the situation, at least nothing will be easy with GAIA, TGAS-RAVE, when all selection functions have to be taken into account, etc.

In the mean time, our knowledge of vertical perturbations, due to satellites, such as the Magellanic clouds, or Sgr dwarf, with masses up to 1/10 of that of the Galaxy, is progressing quickly. There were several talks about these oscillations of the plane, called 
"Galactic Seismology", by A. Sheffield, A. Quillen, L. Widrow, C. Laporte, and F. Gomez. Several stellar "streams" in the outer disk have been interpreted as a manifestation of these oscillations: TriAndromeda, Monoceros Ring, A13. These might be a common perturbation, including the warp and subsequent corrugations of the MW disk (Xu, Newberg et al. 2015).

\section{Cosmological models of the Milky Way}

In her review talk, C. Scannapieco addressed the $\Lambda$ CDM problem of angular momentum, which is being solved. Low angular momentum gas is accreted first, by galaxies with shallow potential well, easy to eject. Then later, higher angular momentum gas is accreted, and forms the disks observed today. It is also possible to form disks with light bulges, in under-dense environments where mergers are rare.

She presented CLUES simulations, made to represent the Local Group formation and to reproduce that of the MW and M31. The main difference between the two galaxies is not the environment, but the merger history is the most important.

About the cusp-core problem, could it be moderated by velocity dispersion? K. Oman proposed that observations are not tracing the cusp, which is present in the DM background in dwarf galaxies, because high gas dispersion washes out the inner peak in the rotation curve. In cosmological simulations of dwarf galaxies (APOSTLE, Oman et al. 2017), they show dwarfs with high non-circular motions. The velocity dispersion has a high impact on measured rotation curves, which then mimic a cored-profile. Real dwarf galaxies show also strong non-circular motions.

Finally, A. Wetzel unveiled in his talk a very welcome chemodynamical success! In the frame of several FIRE simulations, and in particular the Latte project, they aim to simulate the MW formation, and the production of elements: $\mathrm{H}, \mathrm{He}, \mathrm{C}, \mathrm{N}, \mathrm{O}, \mathrm{Ca}$, $\mathrm{Si}, \mathrm{Mg}, \mathrm{Na}, \mathrm{Fe}$. It was possible in 2 cases to obtain a bimodal $[\alpha / \mathrm{Fe}]-[\mathrm{Fe} / \mathrm{H}]$ diagram, like the well known feature of our Galaxy, tracing clearly the thin and thick disks. The gap between the two curves corresponds to a merger at $\mathrm{z} \sim 1$, suddenly quenching star formation and element production, and re-starting a new sequence, with new gas, in line with the so-called two infall model suggested by Chiappini et al. (1997) exactly 20 years ago.

In addition, he showed that the missing satellite problem has received a beginning of solution, in the destruction of satellites close to the Galaxy, when a compact baryonic disk is present: the presence of the galaxy disk at the center eliminates through tidal field many sub-halos within $50 \mathrm{kpc}$ (Garrison-Kimmel, Wetzel et al. 2017).

\section{The future: space for improvement}

We learned during this week many ways to improve our knowledge, I just point towards the main ones:

-(i) About old stars and fossil records of MW formation, better ages will be obtained in the near future, better abundances, larger statistics, and theoretical progress should be made in chemical processes

-(ii) The inner galaxy is not well known, because of distance and obscuration, together with crowding. We need better constraints on the thick disk and the boxy/peanut bulge, the mixture of stellar populations, the nuclear disk or bar.

-(iii) Ages of stars is a domain where progress will be the most spectacular, due to asteroseismology yielding accurate masses. Many surveys by GAIA (T, g, Fe/H), CoRoTGES, RAVE, LSST, and in the long-term Plato will provide exquisite data. 
-(iv) In the domain of the gravitational potential and dynamics of the MW, huge progress will come from GAIA (distances, 3D velocities), which should bring constraints to the dark matter halo, and also on galaxy formation. Cosmological simulations will catch up with more refined baryonic processes.

One special note should be made on the advent of the Big Data era, and the use of deep learning algorithms. The present spectroscopic stellar surveys (high resolution: APOGEE, GALAH, Gaia-ESO, or low resolution: RAVE, LAMOST) and in the near future WEAVE, 4MOST, the photometric surveys (VVV, PanSTARRS, SkyMapper..) and the GAIA revolution, with astrometry and proper motions for a billion stars, all contribute to the deluge of data, requiring new analysis methods.

Deep learning methods have been discussed this week (CANNON, t-SNE) which is a mark that the domain has become mature. The methods are no longer based only on the physical processes, the stellar evolution models. The same occurred in meteorological predictions, and closer to us for instance for the subtraction of foregrounds in Planck data.

Concerning the very near future, the GAIA DR2 release next April, I am sure that you are all in your starting blocks!

Many thanks to Cristina and the organizing committees for such a wonderful week!

\section{References}

Anders, F., Chiappini, C., Rodrigues, T. S. et al. 2017a, A\& A, 597, A30

Anders, F., Chiappini, C., Minchev, I. et al. 2017b, $A \mathscr{E} A$, 600, A70

Athanassoula, E., Rodionov, S. A., Peschken, N., Lambert, J. C.: 2016, ApJ, 821, 90

Athanassoula, E., Rodionov, S. A., Prantzos, N.: 2017 MNRAS, 467 L46

Bensby, T., Feltzing, S., Oey, M. S.: 2014, A\&A, 562, A71

Binney, J., 2017, in IAU Symposium 330, eds. Recio-Blanco, de Laverny \& Brown

Carollo, D., Beers, T. C., Placco, V. M. et al.: 2016 NatPh, 12, 1170

Cescutti, G., Chiappini, C.: 2014, A\&A, 565, A51

Chiappini, C., Anders, F., Rodrigues, T. S. et al.: 2015, AछA, 576, L12

Chiappini, C., Montalban, J., Steffen, M.: 2016, AN, 337, 773

Chiappini, C., Matteucci, F., Gratton, R.: 1997, ApJ, 477, 765

Debattista, V. P., Ness, M., Gonzalez, O. A. et al.: 2017, MNRAS, 469, 1587

Di Matteo, P., Haywood, M., Gomez, A. et al.: 2014 A $\& A$, 567, A122

Fragkoudi, F., Di Matteo, P., Haywood, M. et al 2017, $A \& \& A$, sub (IAU S334, poster)

Fuhrmann, K.: 2011, MNRAS, 414, 2893

Garrison-Kimmel, S., Wetzel, A., Bullock, J. S. et al 2017, MNRAS, sub, (arXiv)

Hayden, M. R., Bovy, J., Holtzman, J. A. et al.: 2015, ApJ, 808, 132

Haywood, M., Di Matteo, P., Lehnert, M. D. et al., 2013, A\&A, 560, A109

Jofré, P., Heiter, U., Worley, C. C. et al.: 2017, A\&A A, 601, A38

Khoperskov, S., Haywood, M., Di Matteo, P., Lehnert, M., Combes, F.: 2017 A $\mathscr{E} A$, sub (IAU S334, poster)

Martig, M., Rix, H-W., Silva Aguirre, V. et al. 2015, MNRAS, 451, 2230

Martig, M., Minchev, I., Ness, M. et al. 2016, ApJ, 831, 139

Meynet, G., Ekström, S., Maeder, A.: 2006, A\& A, 447, 623

Meynet, G., Hirschi, R., Ekström, S. et al.: 2010, A\&A, 521, A30

Miglio, A. et al.: 2017, $A N, 338,644$

Minchev, I., Martig, M., Streich, D. et al.: 2015, ApJ, 804, L9

Minchev, I., Chiappini, C., Martig, M.: 2014, A\&A, 572, A92

Minchev, I., Chiappini, C., Martig, M.: 2016, AN, 337, 944

Oman, K. A., Marasco, A., Navarro, J. F. et al.: 2017, MNRAS, sub (arXiv)

Portail, M., Gerhard, O., Wegg, C., Ness, M.: 2017a, MNRAS, 465, 1621 
Portail, M., Wegg, C., Gerhard, O., Ness, M.: 2017b, MNRAS, 470, 1233

Rahimi, A., Carrell, K., Kawata, D.: 2014, RAA, 14, 1406

Recio-Blanco, A., Rojas-Arriagada, A., de Laverny, P. et al.: 2017, A\& A, 602, L14

Robin, A. C., Bienaymé, O., Fernandez-Trincado, J. G., Reylé, C.: 2017, $A \mathscr{E} A$, in press, (arXiv)

Snaith, O. N., Haywood, M., Di Matteo, P., Lehnert, M., Combes, F., Katz, D., Gomez, A.: 2014, ApJ, 781, L31

Snaith, O. N., Haywood, M., Di Matteo, P., Lehnert, M., Combes, F., Katz, D., Gomez, A.: 2015, A\& $A, 578, \mathrm{~A} 87$

Soderblom, D.R.: 2010, ARA\&A 48, 581

Wegg, C., Gerhard, O., Portail, M.: 2017, ApJL, in press (ArXiv)

Xu, Y., Newberg, H. Jo, Carlin, J. L. et al. 2015, ApJ, 801, 105

Zoccali, M., Vasquez, S., Gonzalez, O. A. et al. 2017, A\&A, 599, A12 